\title{
Generic cuts in models of arithmetic
}

\author{
Richard Kaye \\ 15th February 2007, revised 13th May 2007
}

\begin{abstract}
We present some general results concerning the topological space of cuts of a countable model of arithmetic given by a particular indicator $Y$.

The notion of 'indicator' is defined in a novel way, without initially specifying what property is indicated and is used to define a topological space of cuts of the model. Various familiar properties of cuts (strength, regularity, saturation, coding properties) are investigated in this sense, and several results are given stating whether or not the set of cuts having the property is comeagre.

A new notion of 'generic cut' is introduced and investigated and it is shown in the case of countable arithmetically saturated models $M \vDash \mathrm{PA}$ that generic cuts exist, indeed the set of generic cuts is comeagre in the sense of Baire, and furthermore that two generic cuts within the same 'small interval' of the model are conjugate by an automorphism of the model.

The paper concludes by outlining some applications to constructions of cuts satisfying properties incompatible with genericity, and discussing in model-theoretic terms those properties for which there is an indicator $Y$.
\end{abstract}

\section{Introduction}

For this paper, we look at cuts or initial segments of models of Peano arithmetic (PA), i.e., initial subsets closed under the operations of successor, addition and multiplication. As we have no need to look at subsets not closed under addition or multiplication, the terms 'cut' and 'initial segment' will be in all practical terms synonomous.

This paper presents some general results concerning the topological space of initial segments of a model of arithmetic, $M$, and introduces natural subspaces of cuts given by a particular indicator $Y$. We shall look at this space as a tool for examining constructions of cuts of the model and other structural properties of the model. In particular, we shall look at generic cuts of the model, that is, the class of almost all cuts in the sense of Baire, and describe these; in the case when $M$ is a countable recursively saturated model we give a back-and-forth system that classifies such cuts up to conjugacy.

Indicators were first introduced by Kirby and Paris [3] as a way of constructing initial segments of models of arithmetic. They provide an elegant and rather uniform way of handling most of the main classes of cuts that arise. In due course they also led to independence results for Peano arithmetic [8, 10], and these independence results have been studied extensively and re-proved by 
other means. However indicators are still an important tool for understanding the structural properties of models of arithmetic and their initial segments, and some interesting questions about them remain.

This paper starts by presenting a rather more general study of indicators than is usual. Our first aim is to abstract the main properties of 'an indicator' independently of the set of cuts it is supposed to indicate. Indicators will be used later to isolate the topological subspaces of interest. The goal is to prove results about the structure of the model, the topological properties of the set of its cuts identified by the indicator, and in particular to investigate the complexity of constructions of such cuts. Sections 4 and 5 form the bulk of the paper and is an investigation of the properties of almost all cuts (in the sense of Baire) and the new notion of generic cuts. The final section returns to the question of indicators and the sets of cuts that they indicate.

All terminology used here is standard and except where indicated is as in Models of Peano Arithmetic [1]. For further information, and in particular for information on semiregular, regular and strong cuts, I recommend Kossak and Schmerl's book [4]. Throughout this paper, $\mathscr{L}_{\mathrm{A}}$ is the usual language of arithmetic and $M$ denotes a fixed countable nonstandard model of arithmetic; for sake of definiteness we shall assume $M \vDash \mathrm{PA}$ throughout, though much less than this (such as $M \vDash I \Delta_{0}$ ) suffices for several of our results. The set of elements definable in $M$ by a formula in $\mathscr{L}_{\mathrm{A}}$ with parameters from $C$, i.e., the definable closure of $C=\{\bar{c}\} \subseteq M$ will be denoted $\operatorname{dcl}(\bar{c})$.

For simplicity we restrict our attention to indicators without parameters that work in a uniform way for all $M \vDash \mathrm{PA}$, though other variations such as indicators defined with parameters in a particular model, or more complex indicators for $\Sigma_{n}$-elementary cuts are possible. Similarly, several of our results apply to uncountable models too, but in this respect there are many delicate points here and we leave the topic of uncountable models to future investigations.

\section{Indicators}

A question that has been around for some time, which I first heard asked by Jeff Paris, asks what properties of an initial segment $I$ can be indicated by an indicator. In part, this questions motivates some of the work on indicators discussed here, and some of the results of this paper may be seen to be as a partial answer to this question (Propositions 2.6 and 2.7, Theorem 6.2). The question is presented here as I learnt it; what it actually might mean is discussed later.

Question 2.1 (Paris). Give a preservation theorem for properties of cuts with indicators.

Here, we would like a logical characterisation in syntactic or quasi-syntactic style of those properties that can be described by indicators. The main difficulty with this question is that the same indicator may indicate several different properties. (This phenomenon is called symbiosis by Paris and Kirby. See Definition 6.3.) Some of these properties may be easily described in logical terms (for example, in a first-order way) and others may be considerably harder to describe. 
To progress, we shall be more precise about our terms, 'indicator', etc. Our definition of indicator is novel in that it tries to abstract the properties of an 'indicator' without actually specifying what property is indicated. (But see Proposition 2.6 below which gives at least some property.)

Definition 2.2. Let $M$ be a nonstandard model of arithmetic (always, for this paper, countable and at least $\left.I \Delta_{0}\right)$. An indicator $Y$ is a $\Sigma_{1}$ formula $Y(x, y)=z$ for which the following properties hold, in the model, or provably in the theory, as required.

1. $\forall x \forall y \exists ! z Y(x, y)=z$

2. $x \geqslant y \rightarrow Y(x, y)=0$

3. $x \leqslant x^{\prime}<y^{\prime} \leqslant y \rightarrow Y(x, y) \geqslant Y\left(x^{\prime}, y^{\prime}\right)$

4. If $Y(x, y)>\mathbb{N}$ then $y>(x+1)^{2}$

5. If $Y(x, y)>z$ then there are $x^{\prime}, y^{\prime}$ such that $Y\left(x^{\prime}, y\right)=Y\left(x, y^{\prime}\right)=z$

6. If $Y(x, y)>\mathbb{N}$ and $x<z<y$ then either $Y(x, z)>\mathbb{N}$ or $Y(z, y)>\mathbb{N}$

Example 2.3. There is a 'minimal' indicator $Y_{0}(x, y)$, defined to be the greatest $k$ such that $f^{(k)}(x) \leqslant y$ where $f$ is the function defined by $f(u)=(u+1)^{2}$. This function indicates the presence of initial segments (i.e., without any additional specified property in the sense that $Y(a, b)>\mathbb{N}$ holds iff there is a cut $I \subseteq \mathrm{e} M$ with $a \in I<b)$.

In general, indicators are often defined by $Y(x, y)=\max \left\{k \mid F^{(k)}(x) \leqslant y\right\}$ or $\max \left\{k \mid F_{k}(x) \leqslant y\right\}$, for some increasing function $F$ or family of functions $F_{k}$. Indeed, for each indicator $Y$ in PA there is a family of partial functions, which we will denote $Y_{n}$, defined by $Y_{n}(x)=\min \{y \in M \mid Y(x, y) \geqslant n\}$, and in fact for many natural indicators, these $Y_{n}$ are partial functions even in the weaker base theory I $\Delta_{0}$. (Note that when we write ' $Y(x, y)=z$ ', or any other formula without specifying which model it is interpreted in, we mean that it is true in the ground model $M$, and not necessarily in any initial segment $I$ of $M$; see also Definition 2.8 below.)

We are going to discuss properties of indicators in this generality. The first result is well-known.

Proposition 2.4. Let $Y$ be an indicator. Then for any $x<y \in M$ with $Y(x, y)>\mathbb{N}$ there is $z$ with $x<z<y$ and both $Y(x, z)>\mathbb{N}$ and $Y(z, y)>\mathbb{N}$.

Proof. Let $z$ be least such that $Y(x, z) \geqslant Y(z, y)$. Note that the standard properties of indicators imply that $Y(x, z)>\mathbb{N}$. Also, as $Y(z-1, y)>Y(x, z-1)$ we have $Y(z-1, y)>\mathbb{N}$. Thus by other elementary properties of indicators, $Y(z, y)>\mathbb{N}$ too.

An indicator usually is thought of as indicating the presence of a cut $I$ with a particular property. In general the property of the cut under consideration concerns how the cut sits inside the ground model $M$ and so is more accurately considered as a property of the expanded structure $(M, I)$.

Definition 2.5. An indicator $Y$ indicates cuts with the property $Q^{M}(I)$ if whenever $a<b \in M$ then 
$Y(a, b)>\mathbb{N}$ iff there is a cut $I$ with $a \in I<b$ such that $Q^{M}(I)$.

As the ambient model $M$ is usually fixed for this paper, we shall often write our properties $Q^{M}(I)$ as $Q(I)$, omitting the possible dependence on $M$ from the notation.

Proposition 2.6. Let $Y$ be an indicator. Then $Y$ indicates cuts $I$ with the following property $Q_{Y}(I)$ : 'for all $a \in I$ and $b>I$ we have $Y(a, b)>\mathbb{N}$.'

Proof. One direction is clear: if $Q_{Y}(I)$ holds and $a \in I<b$ then $Y(a, b)>\mathbb{N}$. For the other direction, given $a=a_{0}, b=b_{0}$ with $Y(a, b)>\mathbb{N}$ we can, using the properties of the indicator given above and countability of $M$ choose an $\omega$-sequence of successively smaller intervals $\left[a_{n+1}, b_{n+1}\right] \subseteq\left[a_{n}, b_{n}\right]$ such that $Y\left(a_{n}, b_{n}\right)>\mathbb{N}$ for each $n$ and the sequences $\left(a_{n}\right)$ and $\left(b_{n}\right)$ both tend to some initial segment $I$ as $n \rightarrow \infty$. Then for $x \in I<y$ we must have $x \leqslant a_{n}$ and $b_{n} \leqslant y$ for some $\left[a_{n}, b_{n}\right]$ in the construction so $Y\left(a_{n}, b_{n}\right)>\mathbb{N}$ and hence $Y(x, y)>\mathbb{N}$, as required.

Proposition 2.7. Let $Y$ be an indicator. Then $Y$ indicates cuts $I \subseteq_{\mathrm{e}} M$ such that $I \neq M$ and $P_{Y}(I)$, where $P_{Y}(I)$ is the property: 'for all $a \in I$ and $n \in \mathbb{N}$ there is $b \in I$ such that $Y(a, b)>n$.'

Proof. Let $I \subseteq_{\mathrm{e}} M$ be a proper initial segment which has the property $P_{Y}(I)$. Then given $a \in I$ and $n \in \mathbb{N}$, the least $c \in M$ such that $Y(a, c)>n$ is in $I$. Thus if $b>I$ by properties of the indicator $Y, Y(a, b)>\mathbb{N}$. Thus $I$ has $Q_{Y}(I)$. Conversely, suppose $Q_{Y}(I)$ and $a \in I$ and $n \in \mathbb{N}$. Then for each $b>I$ we have $Y(a, b) \geqslant n$. So by underspill there is some $b \in I$ with $Y(a, b) \geqslant n$ and hence by properties of the indicator, some $b \in I$ with $Y(a, b)=n$.

Given $a, b \in M$ with $Y(a, b)>\mathbb{N}$ there are two obvious cuts $I$ with $a \in I<b$ satisfying $P_{Y}(I)$. The first is $M_{Y}(a)=\{x \in M \mid Y(a, x) \in \mathbb{N}\}$ and the second is $M_{Y}[b]=\{x \in M \mid Y(x, b)>\mathbb{N}\}$. An easy overspill arument shows these cuts are not the same. These cuts generalise the cuts $M(a)$ and $M[b]$ that appear in the literature for certain specific indicators. For example, Kotlarksi [5] studies these for the Paris-Harrington indicator and shows that there are countably many nonisomorphic cuts of the form $M_{Y}(a)$.

Definition 2.8. An indicator $Y$ is absolute if, for all $a, b, c \in M$ and $I \subseteq$ e $M$ containing $a, b, c$ such that $P_{Y}(I)$ holds, $M \vDash Y(a, b)=c$ if and only if $I \vDash$ $Y(a, b)=c$.

One way that $Y$ might be absolute is if the formula $Y(x, y)=z$ is $\Delta_{0}$, rather than just $\Sigma_{1}$. In some cases, if $Y(x, y)=z$ is the formula $\exists \bar{w} \theta(x, y, z, \bar{w})$ with $\theta(x, y, z, \bar{w})$ being $\Delta_{0}$ then we may replace this with the $\Delta_{0}$ formula stating that $z$ is least such that $\exists z^{\prime}, \bar{w} \leqslant z \theta\left(x, y, z^{\prime}, \bar{w}\right)$, and the same cuts will be indicated. Or it may be that the 'witnesses' $\bar{w}$ required in $Y(x, y)=z$ are bound by some $Y_{n}(y)=\min \left\{y^{\prime} \mid Y\left(y, y^{\prime}\right) \geqslant n\right\}$ for some $n \in \mathbb{N}$. Most 'natural' indicators are absolute.

In the case when the indicator $Y$ is absolute, the property $P_{Y}(I)$ depends on $I$ only and not on $(M, I)$. In the sequel we will focus on $P_{Y}(I)$ rather than $Q_{Y}(I)$ because it is more convenient in the case of absolute indicators, and because the special case of the improper initial segment $M$ is often topologically important as a limit of proper initial segments. The proof of the last proposition shows that this is the only initial segment that might have property $P_{Y}$ but not $Q_{Y}$. 


\section{The topological space of indicated cuts}

We start our work proper in this section, by defining for each indicator $Y$ a topological space, denoted $Z_{Y}^{M}$ or more simply as $Z_{Y}$, whose elements are the cuts in $M$ indicated by $Y$.

Definition 3.1. Let $Y$ be an indicator. The set $Z_{Y}$ is the set of (not necessarily proper) $I \subseteq$ e $M$ such that $P_{Y}(I)$ holds. A basic open subset of $Z_{Y}$ is either a set of the form $(a, b)_{Y}=\left\{I \in Z_{Y} \mid a \in I<b\right\}$ for some $a, b \in M$ with $Y(a, b)>\mathbb{N}$, or is a set of the form $(a, \infty)_{Y}=\left\{I \in Z_{Y} \mid a \in I\right\}$. An open subset of $Z_{Y}$ is a union of basic open subsets.

We shall use round-bracket intervals such as $(a, b)_{Y}$ or $(a, \infty)_{Y}$ for sets of cuts, omitting the subscript $Y$ if it is clear from context. In contrast, squarebracket intervals such as $[a, b]$ will always denote sets of elements of $M$, in this case $\{x \in M \mid a \leqslant x \leqslant b\}$. Definition 3.1 makes $Z_{Y}$ into a topological space, as the intersection of two basic open subsets is either empty or a basic open subset. Basic open subsets of the form $(a, \infty)_{Y}$ are only needed if $P_{Y}(M)$ holds; otherwise there will be $b \in M$ such that $(a, \infty)_{Y}=(a, b)_{Y}$.

Proposition 3.2. If $Z_{Y}$ is non-empty, it contains a maximum cut $I_{\max }=\bigcup Z_{Y}$ and a minimum cut $I_{\min }=\bigcap Z_{Y}$.

Proof. Easy.

For the 'minimal' indicator $Y_{0}(x, y)$ of Example 2.3, we denote $Z_{Y_{0}}$ more simply as $Z_{0}$. This space consists of all initial segments of $M$ with the topology given above.

Proposition 3.3. Let $Y$ be an indicator. Then $Z_{Y}$ is a closed subset of the space $Z_{0}$ of all initial segments of $M$, and the topology on $Z_{Y}$ is precisely the subspace topology inherited from $Z_{0}$.

Proof. Let $I \subseteq$ e $M$ satisfy $\neg P_{Y}(I)$. Then there is $a \in I$ such that $Y(a, b) \leqslant n$ for all $b \in I$ and hence by overspill (if $I \neq M$ ) some $b>I$ with $Y(a, b) \leqslant n$. Thus the basic open neighbourhood of $I, U=(a, b)_{0}$ is disjoint to $Z_{Y}$. In the case when $I=M$ we may take $U=(a, \infty)_{0}$ instead, and the argument is the same. If $U \subseteq Z_{Y}$ is open in the subspace topology, then for each $I \in U$ there is a basic open set $V=(a, b)_{0}$ of $Z_{0}$ such that $\left\{J \in Z_{Y} \mid J \in V\right\}$ contains $I$. But then this latter set is precisely the basic open set $(a, b)_{Y}$ of $Z_{Y}$, and so the topologies coincide as stated.

Proposition 3.4. Let $Y$ be an indicator such that $Z_{Y}$ is non-empty. Then $Z_{Y}$ is homeomorphic to the Cantor space $2^{\mathbb{N}}$. In particular, if $a, b \in M$ with $Y(a, b)>\mathbb{N}$ there are $2^{\aleph_{0}}$ initial segments $I$ with $a \in I<b$ and $P_{Y}(I)$.

Proof. Enumerate $M$ as $x_{0}, x_{1}, \ldots$, and define open sets $\left(a_{\sigma}, b_{\sigma}\right)$ of $Z_{Y}$ for $\sigma \in$ $2^{<\omega}$, strings of 0 s and 1 s of finite length. We will arrange that, for all $k, Z_{Y}$ is the disjoint union of all $\left(a_{\sigma}, b_{\sigma}\right)$ as $\sigma$ ranges over strings of length $k$.

To start, we set $\left(a_{\emptyset}, b_{\emptyset}\right)$ to be $(0, \infty)$. Given $\left(a_{\sigma}, b_{\sigma}\right)$ with $\sigma$ of length $k$, we first choose $a_{\sigma 0}=a_{\sigma}<b_{\sigma 0}=a_{\sigma_{1}}<b_{\sigma_{1}}=b_{\sigma}$ using Proposition 2.4 so that $\left(a_{\sigma 0}, b_{\sigma 0}\right)$ and $\left(a_{\sigma 1}, b_{\sigma 1}\right)$ are both non-empty. In particular, if $x_{k}$ is a suitable choice for $b_{\sigma 0}=a_{\sigma 1}$ we use this. Otherwise we choose some other value. Now 
consider the other end-points, $\left(a_{\sigma 0}\right.$ and $\left.b_{\sigma 1}\right)$. If $x_{k}$ was not used for $b_{\sigma 0}=a_{\sigma_{1}}$ then we may be able to replace one of $\left(a_{\sigma 0}\right.$ and $\left.b_{\sigma 1}\right)$ with $x_{k}$ and having the set of $I \in Z_{Y}$ so defined stay the same; if we can do this then we do.

The construction results in a full binary tree of intervals such that each infinite path through the tree has a unique limit in $Z_{Y}$. (The uniqueness of the limit is from the way we used the enumeration of the model and used $x_{k}$ whenever possible as an end-point.) Therefore this gives an homeomorphism between $Z_{Y}$ and the Cantor space.

\section{Almost all cuts in the sense of Baire}

In this section we shall fix some countable model $M$ and an indicator $Y$ such that $Z_{Y}$ is non-empty.

Definition 4.1. An interval (or $Y$-interval) of $M$ is a set $[a, b]=\{x \in M \mid a \leqslant$ $x \leqslant b\}$ such that $a<b$ and $Y(a, b)>\mathbb{N}$.

If $[a, b]$ is an interval and $I \in Z_{Y}$ is an initial segment of $M$ we shall write (with slight abuse of notation) ' $I \in[a, b]$ ' for the statement ' $a \in I$ and $b \notin I$ '. In this case we say ' $[a, b]$ contains $I$ '.

As already noted, the space $Z_{Y}$ is isomorphic to the Cantor set and so is compact and Hausdorff. Therefore Baire's theorem applies. We shall study properties of 'almost all' $I \in Z_{Y}$ in the sense of Baire.

The most natural construction of initial segments $I \in Z_{Y}$ is by the successiveinterval technique used above in Proposition 2.6. This corresponds in the topological setting to building a cut in $Z_{Y}$ by a Banach-Mazur game [7, Chapter 6], i.e., by looking at comeagre subsets of $Z_{Y}$. For this section we look at such subsets of $Z_{Y}$ or (equivalently) cuts $I \in Z_{Y}$ constructed by the Banach-Mazur game.

Definition 4.2. A subset $S \subseteq Z_{Y}$ (or property $P(I)$ ) is enforceable by $Y$ iff the set $S$ (or the set of $I \in Z_{Y}$ satisfying $P(I)$ ) is comeagre in $Z_{Y}$.

By Baire's theorem and the definition of a comeagre set, any enforceable property has continuum-many cuts satisfying it, and these cuts are dense in $Z_{Y}$. Moreover, the intersection of any countable number of enforceable subsets is enforceable and hence non-empty.

We now give a sequence of results concerning enforceable properties. The first of these is obtained by modifying an argument due to Paris (written up by Kotlarski [5, theorem 3]) that the cuts $I \vDash \mathrm{PA}$ form a meagre subset of $Z_{\mathrm{PH}}$, where $Y_{\mathrm{PH}}$ is the Paris-Harrington indicator for PA.

Theorem 4.3. It is enforceable that $\{n \in M \mid \forall x \in I \exists y \in I Y(x, y) \geqslant n\}=\mathbb{N}$.

Proof. Let $\alpha>\mathbb{N}$ in $M$ be nonstandard and let

$$
A_{\alpha}=\left\{I \in Z_{Y} \mid \exists x \in I \forall y \in I Y(x, y)<\alpha\right\} .
$$

We show that $A_{\alpha}$ is open and dense. The result then follows from the countability of $M$ and looking at the comeagre set $\bigcap_{\alpha>\mathbb{N}} A_{\alpha}$. But given non-empty open $(a, b) \subseteq Z_{Y}$ with $Y(a, b)>\mathbb{N}$ we may simply choose $c \leqslant b$ maximal so that $Y(a, c)<\alpha$. As $Y(a, b)>\mathbb{N}$ and $\alpha>\mathbb{N}$ the set $(a, c) \subseteq(a, b)$ is open and non-empty as required. 
Corollary 4.4. It is enforceable that the cut $I$ is not strong.

Proof. (Sketch.) Let $I$ be as in the previous result and working in $M$ define $f(\langle x, n\rangle)$ to be the least $y$ such that $Y(x, y) \geqslant n$, or some fixed $b>I$ if no such $y$ exists. Then by overspill there cannot be $c \in M$ such that, for all $x, n \in I$, $f(\langle x, n\rangle) \in I$ iff $f(\langle x, n\rangle)<c$; this shows $I$ is not strong in $M$.

Corollary 4.5. Let $Y$ be absolute. Then it is enforceable that:

1. $\mathbb{N}$ is $\Pi_{2}$-definable (without parameters) in $I$;

2. $I \not \forall \mathrm{I} \Sigma_{2}$;

3. $I$ is not $\Pi_{2}$-recursively saturated.

Proof. All of these follow from the previous theorem and absoluteness, which shows that there is a $\Pi_{2}$ formula $\theta(x)$ (namely $\forall y \exists z Y(y, z) \geqslant x$ ) such that it is enforceable that $\mathbb{N}$ is defined by this formula in $I$. The last two properties listed follow since every model of $\mathrm{I} \Sigma_{2}$ satisfies the least number principle for $\Sigma_{2}$ formulas [9], and the recursive $\Pi_{2}$ type $\{\theta(x)\} \cup\{x>n \mid n \in \mathbb{N}\}$ is not realised in $I$.

Given that it is enforceable that $\mathbb{N}$ is $\Pi_{2}$-definable it is natural to ask if it is also enforceable that $\mathbb{N}$ is $\Sigma_{2}$-definable. The answer to this question is 'no'. In fact, slightly more is true.

Theorem 4.6. It is enforceable that $I$ is $\Sigma_{2}$-recursively saturated as an $\mathscr{L}_{\mathrm{A}^{-}}$ structure.

Proof. We play a step of the Banach-Mazur game with some non-empty interval $(a, b)_{Y}$ given, considering a recursive set of formulas

$$
p(x)=\left\{\exists \bar{y} \forall \bar{z} \theta_{i}(x, \bar{y}, \bar{z}) \mid i \in \mathbb{N}\right\}
$$

where each $\theta_{i}(x, \bar{y}, \bar{z})$ is $\Delta_{0}$. The formulas in $p(x)$ may involve any finite set of parameters from $[0, a]$. We will choose non-empty $\left(a^{\prime}, b^{\prime}\right)_{Y} \subseteq(a, b)_{Y}$ such that either $p(x)$ is not finitely satisfied in all $I \in\left(a^{\prime}, b^{\prime}\right)_{Y}$ or else $p(x)$ is satisfied in all $I \in\left(a^{\prime}, b^{\prime}\right)_{Y}$. By using a pairing function we may assume each $\bar{y}, \bar{z}$ is a single variable, and by using another pairing function and considering $\langle x, y\rangle$ we may assume that the quantifier $\exists y$ is absent and our set of formulas is $p(x)=\left\{\forall z \theta_{i}(x, z) \mid i \in \mathbb{N}\right\}$.

We are done if we can find $a \leqslant a^{\prime}<b^{\prime} \leqslant b$ with $Y\left(a^{\prime}, b^{\prime}\right) \geqslant n$ for each $n \in \mathbb{N}$ and $\exists x<a^{\prime} \bigwedge_{i<n} \forall z<b^{\prime} \theta_{i}(x, z)$ for each $n \in \mathbb{N}$. For then by saturation in $M$ there is $x<a^{\prime}$ with $\forall z<b^{\prime} \theta_{i}(x, z)$ for all $i$ and thus $p(x)$ is reaslied in any $I \in\left(a^{\prime}, b^{\prime}\right)_{Y}$ since each $\theta_{i}$ is $\Delta_{0}$. Otherwise, there is $n \in \mathbb{N}$ such that

$$
M \vDash \forall a^{\prime}, b^{\prime}\left(a \leqslant a^{\prime}<b^{\prime} \leqslant b \wedge Y\left(a^{\prime}, b^{\prime}\right) \geqslant n \rightarrow \forall x<a^{\prime} \bigvee_{i<n} \neg \forall z<b^{\prime} \theta_{i}(x, z)\right)
$$

Now let $I \in(a, b)_{Y}$ satisfy $P_{Y}(I)$. Then for any $x<a^{\prime} \in I$ there is $b^{\prime} \in I$ with $Y\left(a^{\prime}, b^{\prime}\right) \geqslant n$ and hence some $z<b^{\prime}$ and $i<n$ such that $M \vDash \neg \theta_{i}(x, z)$. Thus by absoluteness of $\Delta_{0}$ formulas $p(x)$ is not finitely satisfied in $I$.

This step is played in the Banach-Mazur game for all such sets $p(x)$ of formulas in parameters from the constructed cut $I$. 
In fact, the previous result can be improved by adding to the language of $I$ subsets of $I$ coded in $M$, and showing that any recursive $\Sigma_{2}$ type involving finitely many of these parameters is realised. The details are left as an exercise.

The special case of countable short $\Pi_{1}$-recursively saturated $\mathscr{L}_{\mathrm{A}}$-structures $I$ is worth noting, as this property is useful for constructing end-extensions. A model $I$ is short $\Pi_{1}$-recursively saturated if, for every recursive set $p(x)$ of formulas in $\mathscr{L}_{\mathrm{A}}$ with finitely many parameters from $I$, including one parameter $a$ such that $x<a$ is in $p(x)$, we have: $p(x)$ is realised iff it is finitely satisfied.

Proposition 4.7. Let $I \vDash \mathrm{B} \Sigma_{1}$ be countable and nonstandard and short $\Pi_{1}$ recursively saturated. Suppose $T=T(\bar{a})$ is a coded set of sentences in $\mathscr{L}_{\mathrm{A}} \cup\{\bar{a}\}$ where $\bar{a} \in I$ are finitely many parameters, such that $I \vDash \Pi_{1}-\operatorname{Th}(T)$, the set of $\Pi_{1}$ consequences of $T$. Then there is an end-extension $J \supseteq_{\mathrm{e}} I$ such that $J \vDash T$.

Results of this type were given by Paris and Wilkie [11]. This particular one can be proved by inductively constructing a complete extension $T_{c}=T \cup$ $\left\{\sigma_{0}, \sigma_{1}, \ldots\right\}$ in the language $\mathscr{L}_{\mathrm{A}} \cup I$ such that $I \vDash \Pi_{1}-\operatorname{Th}\left(T \cup\left\{\sigma_{0}, \sigma_{1}, \ldots, \sigma_{n}\right\}\right)$ for each $n$ and for each $b \in I$ the set of formulas $q_{b}(x)=\{x<b\} \cup\{x \neq c \mid c \in I\}$ has no support. Thus by the omitting types theorem there is a model $J$ of $T_{c}$ omitting all $q_{b}(x)$ and $J$ can be regarded as a (not necessarily proper) endextension of $I$.

Obviously a $\Sigma_{2}$-recursively saturated model is short $\Pi_{1}$-recursively saturated. Another consequence of $\Sigma_{2}$-recursive saturation is given in the next corollary.

Corollary 4.8. It is enforceable that $\mathbb{N}$ is not $\Sigma_{2}$-definable in $I$ with parameters.

Proof. If $\mathbb{N}=\{n \in I \mid I \vDash \psi(n)\}$ with $\psi(x) \Sigma_{2}$ then the recursive $\Sigma_{2}$ type $p(x)=\{x>n \mid n \in \mathbb{N}\} \cup\{\psi(x)\}$ is finitely satisfied but not reaslised in $I$.

Definition 4.9. A cut $I$ is an $\omega$-limit if in the obvious sense of 'limit', $I=$ $\lim _{n \in \omega}(a)_{n}$ for some monotonic sequence, $(a)_{n}$, of elements of $M$ that is coded in $M$.

Theorem 4.10. It is enforceable that $I$ is not an $\omega$-limit.

Proof. Given $r<s$ with $Y(r, s)>\mathbb{N}$ and $(a)_{n}$, choose $t$ with $Y(r, t)>\mathbb{N}$ and $Y(t, s)>\mathbb{N}$ by Proposition 2.4 and note that by monotonicity at most one of the intervals $[r, t],[t, s]$ contains all but finitely many $(a)_{n}$. Choose some $[r, t]$ or $[t, s]$ not containing some such inifinite set of $(a)_{n}$ s. Repeat this for all $a \in M$.

Paris and Kirby [3] construct cuts satisfying $\mathrm{I} \Sigma_{1}$ via the notion of semiregular cuts. Their construction is a Banach-Mazur game and so (for the right indicator) the property of being semiregular is enforceable. We will look into this argument in slightly greater generality in a moment. On the other hand. the usual constructions of regular cuts, such as the game-construction given by Paris and Kirby in the same paper, are rather more complicated. It seems natural to attempt to prove that it is not enforceable that a cut is regular. For technical reasons, we start with the case of semiregular cuts.

Definition 4.11. Let $Y$ be an indicator and recall the function $Y_{n}$ defined in $M$ by $Y_{n}(x)=\min \{y \in M \mid Y(x, y) \geqslant n\}$. We iterate these functions $Y_{n}$ in the style of the Grzegorczyk hierarchy, defining $\hat{Y}_{n}(x)$ by $\hat{Y}_{0}(x)=Y_{0}(x)$ and, for $n>0$, $\hat{Y}_{n}(x)=\max \left(\hat{Y}_{n-1}^{(x)}(x), Y_{n}(x)\right)$. We also set $\hat{Y}(x, y)=\max \left\{n \mid \hat{Y}_{n}(x) \leqslant y\right\}$. 
Definition 4.12. Two indicators $Y_{1}, Y_{2}$ have the same strength if and only if, for all $a, b \in M, Y_{1}(a, b)>\mathbb{N} \Leftrightarrow Y_{2}(a, b)>\mathbb{N}$.

Theorem 4.13. It is enforceable that $I$ is semiregular if and only if $Y$ and $\hat{Y}$ have the same strength.

Proof. We start by assuming that $Y$ and $\hat{Y}$ do not have the same strength, and there is $[a, b]$ with $Y(a, b)>\mathbb{N}$ but $\hat{Y}(a, b)=n \in \mathbb{N}$. We claim that no $I \in Z_{Y}$ with $a \in I<b$ can be semiregular. Indeed, for some $k<n$ we must have $I$ closed under $\hat{Y}_{k}(x)$ but not under $\hat{Y}_{k+1}(x)$. Let $c \in I$ with $Y_{k+1}(c)>I$ and define $f(x)=Y_{k}^{(x)}(c)$ for $x<c$. This $f$ has $I \cap \operatorname{Im}(f) \subseteq_{\mathrm{cf}} I$, and hence $I$ is not semiregular. Therefore the property of being semiregular is not enforceable, as if such an interval $[a, b]$ exists, our opponent may play it on his first move and there is nothing we can do to build a semiregular cut in the interval $[a, b]$.

On the other hand, if $Y$ and $\hat{Y}$ have the same strength we may as well use the indicator $\hat{Y}$ in place of $Y$. Now given an interval $[a, b]$ with $\hat{Y}_{n}(a) \leqslant b$ for some $n>\mathbb{N}$, and considering some definable function $f:\{x \in M \mid x<c\} \rightarrow M$ where $c<a-1$, let $a_{0}=a$ and $a_{i+1}=\hat{Y}_{n-1}\left(a_{i}\right)$. Then $\left\{a=a_{0}, a_{1}, \ldots, a_{a}\right\} \subseteq[a, b]$ and the interior of at least one of the intervals $\left[a_{i}, a_{i+1}\right]$ must be disjoint with $\operatorname{Im} f$. We play such an interval $\left[a_{i}, a_{i+1}\right]$ in the Banach-Mazur game, dealing with all such definable functions in the same way at some other stage of the game. In this way we enforce the resulting cut to be semiregular.

Remark 4.14. Of course, if $I$ is semiregular then it satisfies $I \Sigma_{1}$, so if $Y$ and $\hat{Y}$ have the same strength then it is enforceable that $I \vDash \mathrm{I} \Sigma_{1}$. The proof just given also shows that if $Y$ is absolute and $Y$ and $\hat{Y}$ do not have the same strength then it is not enforceable that $I \vDash I \Sigma_{1}$.

In contrast with the last theorem, the property of being regular is never enforceable.

Theorem 4.15. It is enforceable that $I$ is not regular. In fact, if $Y$ is absolute, it is enforceable that $I$ does not satisfy $\mathrm{B} \Sigma_{2}$.

Proof. We show how to play the Banach-Mazur game to enforce a failure of regularity. Without serious loss of generality, we may assume that $\hat{Y}$ and $Y$ have the same strength; for if otherwise either we are given $[a, b]$ with $\hat{Y}(a, b) \in \mathbb{N}$ in which case all constructed cuts fail to be semiregular and hence fail to be regular, or else we may play an interval $[c, d] \subseteq[a, b]$ with $\hat{Y}(c, d) \in \mathbb{N}$. We shall assume this is not possible, that is: we are given $[a, b]$ such that $\hat{Y}(c, d)>\mathbb{N}$ for all subintervals $[c, d]$.

We may assume that $b=\hat{Y}_{\mu}(a)$ for some nonstandard $\mu<a-1$ and we start by defining a ' $\mu$-colouring' of $[a, b]$, i.e., a recursive function $F:[a, b] \rightarrow$ $\{0,1, \ldots, \mu-1\}$. The definition of $F$ is by induction on $\mu$.

Suppose $C \subseteq\{0,1, \ldots, \mu-1\}$ and $\left[a_{0}, b_{0}\right]$ is an interval. We define a function $F_{\left[a_{0}, b_{0}\right]}^{C}:\left[a_{0}, b_{0}\right] \rightarrow C$ as follows. If $C=\{c\}$ has a single element, then $F_{\left[a_{0}, b_{0}\right]}^{C}$ is the constant function $F_{\left[a_{0}, b_{0}\right]}^{C}(x)=c$. Otherwise let $n=\operatorname{card} C$ and $C=$ $\left\{c_{0}, c_{1}, \ldots, c_{n-1}\right\}$ in increasing order. and define $a_{i}$ for $i>0$ by $a_{i}=\max \{b \mid$ $\left.\hat{Y}\left(a_{i-1}, b\right) \leqslant n\right\}$ define $C_{i} \subseteq C$ by $C_{i}=C \backslash\left\{c_{i \bmod n}\right\}$ and inductively set $F_{\left[a_{0}, b_{0}\right]}^{C}(x)=F_{\left[a_{i}, a_{i+1}\right]}^{C_{i}}(x)$ whenever $a_{i} \leqslant x<a_{i+1}$. This function $F$ is recursive and given by a $\Sigma_{1}$ formula $F(x)=s$, as the reader may verify. 
We are now going to play the game, constructing a cut $I$ so that for no colour $s<\mu$ are the elements with colour $s$ cofinal in $I$. This obviously means $I$ is not regular, but if $Y$ is absolute (and hence $\hat{Y}$ is absolute) then it also means that $I$ fails to satisfy the following instance of $\mathrm{B} \Sigma_{2}$ :

$$
\forall s<\mu \exists x \forall y>x F(y) \neq s \rightarrow \exists t \forall s<\mu \exists x<t \forall y>x F(y) \neq s .
$$

At a later stage in the game we are given an interval $[r, s] \subseteq[a, b]$, with $\hat{Y}(r, s)=\nu$ and $\mathbb{N}<\nu<\mu$, and we are considering colour $c$. (There are countably many colours, and we consider each at some stage.) At this stage in the construction it is already decided that $r \in I$ and $s>I$. If no $x \in[r, s]$ has $F(x)=c$ there is nothing to do!. Otherwise we may find an interval $\left[a_{i}, a_{i+1}\right] \subseteq$ $[r, s]$ and set of colours $C_{i} \subseteq\{0,1, \ldots, \mu-1\}$ as in some stage of the recursive definition of $F$ such that $F$ is equal to $F_{\left[a_{i}, a_{i+1}\right]}^{C_{i}}$ on $\left[a_{i}, a_{i+1}\right]$, and $\operatorname{card} C_{i}=n$. Then our colour $c$ is some $c_{k}$ in the set $C_{i}$, and in the next level of the definition of $F$ we divide $\left[a_{i}, a_{i+1}\right]$ up into subintervals $\left[a_{i 0}, a_{i 1}\right],\left[a_{i 1}, a_{i 2}\right],\left[a_{i 2}, a_{i 3}\right], \ldots$ where $a_{i j}=\max \left\{b \mid \hat{Y}\left(a_{i j-1}, b\right) \leqslant n\right\}$ and define $F$ as $F_{\left[a_{i j}, a_{i j+1}\right]}^{C_{i} \backslash\left\{c_{j}\right\}}$ on $\left[a_{i j}, a_{i j+1}\right]$. Therefore we can achieve our objective in the game by playing the subinterval $\left[a_{i k}, a_{i k+1}\right]$ for which $F=F_{\left[a_{i k}, a_{i k+1}\right]}^{C_{i} \backslash\left\{c_{k}\right\}}$ does not use colour $c=c_{k}$. We need only check that this is a valid move ni the game, i.e., $k$ is not too big and $\left[a_{i k}, a_{i k+1}\right] \subseteq[r, s]$; but this is clear as $k<\mu<a-1<a_{i k}$ and from the iteration scheme in the definition of $\hat{Y}$.

At this point I shall digress briefly to mention an interesting technical question I was unable to settle.

Question 4.16. Is there a model $I$ of $\mathrm{B} \Sigma_{2}$ such that the set of $x$ in $M$ such that $M \vDash \forall a \quad F_{x}(a)$ exists is precisely the standard cut?

This question appears quite interesting as it asks about the 'height' of ' $n$ dense sets' (i.e., the sets used in the construction of regular cuts in Chapter 4 of Kirby's Ph.D. thesis [2]) or equivalently for information about a stage of the game used in the Paris-Kirby game-construction of regular cuts [3]. It seems a natural idea to settle this by introducing another step in the game-construction to make these sets short. However, I was not able to prove this modification works, and note that the analogous attempt of a modification of the construction of a strong cut cannot work.

We now look at the theory of the cut $I$, and in particular whether it is coded. The following result shows that the $\Pi_{1}$ theory of the cut, with a parameter, is coded.

Theorem 4.17. It is enforceable that for each $a \in I$ the set $\Pi_{1}-\operatorname{Th}(I, a)$ of $\Pi_{1}$ statements true in $I$ is coded in $M$.

Proof. Given $a \in M$ and $r<s$ with $Y(r, s)>\mathbb{N}$, choose $r \leqslant r^{\prime}<s^{\prime} \leqslant s$ with $Y\left(r^{\prime}, s^{\prime}\right)>\mathbb{N}$ and

$$
\exists x<s^{\prime} \theta(x, a) \leftrightarrow \exists x<r^{\prime} \theta(x, a)
$$

for all $\Delta_{0}$ formulas $\theta$. The existence of such $r^{\prime}, s^{\prime}$ is a simple saturation argument in $M$, using Proposition 2.4 to show that infinitely many suitable disjoint subintervals $\left[r^{\prime}, s^{\prime}\right] \subseteq[r, s]$ exist, and therefore for any finite set of $\theta$ s suitable $\left[r^{\prime}, s^{\prime}\right]$ can be found. But having chosen such $\left[r^{\prime}, s^{\prime}\right]$, if $r^{\prime} \in I<s^{\prime}$ then 
$\Pi_{1}-\operatorname{Th}(I, a)=\left\{\forall x \theta(x, a) \mid M \vDash \forall x<s^{\prime} \theta(x, a)\right\}$ and hence this set is coded in $M$. The result follows by using the same strategy on all $a \in M$.

Question 4.18. Assuming if necessary that the indicator $Y$ is absolute, is it enforceable that $\Pi_{2}-\operatorname{Th}(I)$ is not coded in $I$ ?

\section{$5 \quad$ Generic cuts}

Throughout this section we work with an indicator $Y$ and a countable arithmetically saturated model $M \vDash \mathrm{PA}$. (A model $M$ is arithmetically saturated if and only if it is recursively saturated and the standard cut is strong in $M$. For further information on arithmetically saturated models of PA see Kossak and Schmerl [4].) We assume $Z_{Y}$ is non-empty. Our objective is to identify a single property of cuts that is enforceable and implies all other enforceable properties of cuts. We call cuts satisfying this property generic. Analogous results have been proved for different topologies, especially in the context of automorphisms of models. For models of arithmetic, such a characterization of generic automorphisms was achieved by Lascar [6], and we have used this as a model for our results. The context here is, however, quite different and our presentation is self-contained and does not require any of Lascar's results.

We need some definitions.

Definition 5.1. Let $\bar{c} \in M$ be a tuple of finitely many parameters. An interval $[a, b]$ is $\bar{c}$-small for $Y$ (or just $\bar{c}$-small if $Y$ is understood) if for $n=Y(a, b)$ we have $n<x$ for each nonstandard $x \in \operatorname{dcl}(a, \bar{c})$ and, moreover, that $b$ is the least element of $M$ such that $Y(a, b)=n$ for this $n$.

The existence of $\bar{c}$-small intervals is an easy consequence of the arithmetic saturation of $M$.

Proposition 5.2. Let $[a, b]$ be an interval and $\bar{c} \in M$. Then there is a $\bar{c}$-small interval $\left[a^{\prime}, b^{\prime}\right] \subseteq[a, b]$.

Proof. Let $a^{\prime}=a$. We must choose $b^{\prime} \in[a, b]$ such that $Y\left(a^{\prime}, b^{\prime}\right)>\mathbb{N}$ and $\left[a^{\prime}, b^{\prime}\right]$ is $\bar{c}$-small. Let $n=Y(a, b) \in M$ and using the arithmetic saturation of $M$ let $n^{\prime}<n$ be nonstandard such that $n^{\prime}<d$ for each nonstandard $d \in$ $\operatorname{dcl}\left(a^{\prime}, \bar{c}\right)$. Finally, let $b^{\prime}=Y_{n^{\prime}}\left(a^{\prime}\right)$ and the interval $\left[a^{\prime}, b^{\prime}\right]$ is the required $\bar{c}$-small subinterval.

Definition 5.3. Let $\bar{c} \in M$. An interval $[a, b]$ is $\bar{c}$-constant for $Y$ (or just $\bar{c}$-constant if $Y$ is understood) if we have:

Whenever $x \in[a, b]$ and $\left[a^{\prime}, b^{\prime}\right] \subseteq[a, b]$ is a subinterval, then there is $x^{\prime} \in\left[a^{\prime}, b^{\prime}\right]$ such that, for all formulas $\theta(x, \bar{c})$ of $\mathscr{L}_{\mathrm{A}}$ involving parameters $\bar{c}$, we have $M \vDash \theta(x, \bar{c}) \leftrightarrow \theta\left(x^{\prime}, \bar{c}\right)$.

Proposition 5.4. Let $\left[a_{0}, b_{0}\right] \subseteq M$ be an interval and $\bar{c} \in M$. Then there is a subinterval $[a, b] \subseteq\left[a_{0}, b_{0}\right]$ which is $\bar{c}$-constant.

Proof. Given an interval $[r, s] \subseteq\left[a_{0}, b_{0}\right]$, and a formula $\theta(x, \bar{c})$, and trying to find $\left[r^{\prime}, s^{\prime}\right] \subseteq[r, s]$ satisfying the definition for the formula $\theta(x, \bar{c})$, we define $f_{\theta}(r, s)$ to be the greatest $n \in M$ such that there are $r^{\prime}, s^{\prime} \in[r, s]$ with $s^{\prime}=Y_{n}\left(r^{\prime}\right)$ and

$$
\forall x\left(r^{\prime} \leqslant x \leqslant s^{\prime} \rightarrow \neg \theta(x, \bar{c})\right) .
$$


There are two cases. Either $f_{\theta}(r, s)$ is nonstandard, in which case $\left[r^{\prime}, s^{\prime}\right]$ is an interval which satisfies the definition for $\theta(x, \bar{c})$, or else $f_{\theta}(r, s) \in \mathbb{N}$, in which case $[r, s]$ satisfies the condition already. This argument enables us to deal with a single $\theta(x, \bar{c})$. Repeating this argument with different $\theta(x, \bar{c})$ and sucessively refining our interval enables us to deal with any finite number of formulas. To deal with all $\theta(x, \bar{c})$ simultaneously we use arithmetic saturation to encode the construction tree and find an infinite path through this tree.

For each $\theta(x, \bar{c})$, define $a_{\theta}(r, s)$ to be the least $a \in[r, s]$ such that for $n=$ $f_{\theta}(r, s)$ and $b=Y_{n}(a)$ we have $\forall x(a \leqslant x \leqslant b \rightarrow \neg \theta(x, \bar{c}))$. Also, let $b_{\theta}(r, s)$ be this $b$.

Now fix a recursive (and hence coded) enumeration of $\mathscr{L}_{\mathrm{A}}$-formulas $\theta_{0}, \theta_{1}, \theta_{2}, \ldots$ We define functions $f: 2^{<\omega} \rightarrow M, a: 2^{<\omega} \rightarrow M, b: 2^{<\omega} \rightarrow M$, with arguments that a finite sequences of $0 \mathrm{~s}$ and $1 \mathrm{~s}$, as follows. For the empty sequence we set $a(\emptyset)=a_{0}, b(\emptyset)=b_{0}$ corresponding to our initial given interval $\left[a_{0}, b_{0}\right]$, and $f(\emptyset)=f_{\theta_{0}}\left(a_{0}, b_{0}\right)$. Also, assuming that $f(\varepsilon), a(\varepsilon), b(\varepsilon)$ are defined and $\varepsilon$ has length $l$, we set

$$
a(\varepsilon 0)=a_{\theta}(a(\varepsilon), b(\varepsilon)), b(\varepsilon 0)=b_{\theta}(a(\varepsilon), b(\varepsilon)),
$$

and

$$
f(\varepsilon 0)=f_{\theta}(a(\varepsilon), b(\varepsilon)),
$$

where $\theta$ is $\theta_{l+1}$, corresponding to the choice of 'best' subinterval $[a(\varepsilon 0), b(\varepsilon 0)] \subseteq$ $[a(\varepsilon), b(\varepsilon)]$ making $\theta_{l+1}(x, \bar{c})$ false everywhere. Also, set

$$
a(\varepsilon 1)=a(\varepsilon), b(\varepsilon 1)=b(\varepsilon) \text {, and } f(\varepsilon 1)=f(\varepsilon)
$$

corresponding to the choise of selecting the whole interval from $[a(\varepsilon), b(\varepsilon)]$.

These functions, and in particular the function $f: 2^{<\omega} \rightarrow M$, can be encoded in $M$ as $f: \mathbb{N} \rightarrow M$. Using the strength of $\mathbb{N}$ in $\mathrm{M}$ we take $d \in M$ such that for each $\varepsilon \in 2^{<\omega}$ we have:

$$
f(\varepsilon) \in \mathbb{N} \text { if and only if } f(\varepsilon)<d .
$$

Now using the saturation of $M$ we select a coded sequence of intervals

$$
\left[a_{0}, b_{0}\right] \supseteq\left[a_{1}, b_{1}\right] \supseteq\left[a_{2}, b_{2}\right] \supseteq\left[a_{3}, b_{3}\right] \supseteq \ldots
$$

and a coded sequence

$$
\varepsilon_{0}, \varepsilon_{1}, \varepsilon_{2}, \varepsilon_{3}, \ldots
$$

of elements of $2^{<\omega}$ (where $\varepsilon_{i}$ has length $i$, so $\varepsilon=\emptyset$ ) such that, for each $l$,

$$
\text { if } f\left(\varepsilon_{l}\right) \geqslant d \text { then } \varepsilon_{l+1}=\varepsilon_{l} 0 \text {, and }\left[a_{l+1}, b_{l+1}\right]=\left[a\left(\varepsilon_{l} 0\right), b\left(\varepsilon_{l} 0\right)\right]
$$

corresponding to the choice of a part of the interval making the formula false, and

$$
\text { if } f\left(\varepsilon_{l}\right)<d \text { then } \varepsilon_{l+1}=\varepsilon_{l} 1 \text {, and }\left[a_{l+1}, b_{l+1}\right]=\left[a\left(\varepsilon_{l} 1\right), b\left(\varepsilon_{l} 1\right)\right]
$$

for the choice of the whole interval.

Clearly $\left[a_{l}, b_{l}\right]$ has $Y\left(a_{l}, b_{l}\right)>\mathbb{N}$ for each $l$, so by overspill there is $l>\mathbb{N}$ such that

$$
\left[a_{l}, b_{l}\right] \subseteq\left[a_{l^{\prime}}, b_{l^{\prime}}\right] \text { for all } l^{\prime} \leqslant l
$$

and $Y\left(a_{l}, b_{l}\right)>l>\mathbb{N}$. Note too that for each $\theta(x, \bar{c})$, this formulas is $\theta_{i}(x, \bar{c})$ for some $i$ so: 
either $\forall x \in\left[a_{l}, b_{l}\right] \neg \theta_{i}(x, \bar{c})$;

or there is some $n \in \mathbb{N}$ such that between any $a, b \in\left[a_{l}, b_{l}\right]$ with $b=Y_{n}(a)$ there is some $x$ satisfying $\theta_{i}(x, \bar{c})$.

Thus $\left[a_{l}, b_{l}\right]$ satisfies the requirement in Definition 5.3.

To satisfy requirement (a) simply find $c_{l} \in\left[a_{l}, b_{l}\right]$ such that both $\left[a_{l}, c_{l}\right]$ and $\left[c_{l}, b_{l}\right]$ are intervals by Proposition 2.4, and take $\left[c_{l}, b_{l}\right]$. For then if $\exists x<b_{l} \theta(x, \bar{c})$ but not $\exists x<a_{l} \theta(x, \bar{c})$ then there is some $x<Y_{n}\left(a_{l}\right)$ with $\theta(x, \bar{c})$ for some $n \in \mathbb{N}$ by the construction above, and hence $\exists x<c_{l} \theta(x, \bar{c})$.

Here is the definition of a generic cut.

Definition 5.5. A cut $I \in Z_{Y}$ is generic for $Y$ (or generic, if $Y$ is understood) if for all $\bar{c} \in M$ :

(a) there is a $\bar{c}$-small $\bar{c}$-constant interval $[a, b] \subseteq M$ containing $I$;

(b) whenever $[a, b]$ is $\bar{c}$-constant, and contains $I$, and $[r, s] \subseteq[a, b]$ is a $\bar{c}$-small subinterval, then there is $\left[r^{\prime}, s^{\prime}\right] \subseteq[a, b]$ containing $I$ and an automorphism $g \in \operatorname{Aut}(M, \bar{c})$ (i.e., fixing $\bar{c}$ pointwise) such that $g[r, s]=\left[r^{\prime}, s^{\prime}\right]$.

Theorem 5.6. Let $\bar{c} \in M$ and $I, I^{\prime} \in[a, b]$ be generic, where $[a, b]$ is $\bar{c}$-small and $\bar{c}$-constant. Then there is an automorphism $g \in \operatorname{Aut}(M, \bar{c})$ such that $g I=I^{\prime}$.

Proof. We build a suitable $g \in \operatorname{Aut}(M, \bar{c})$ by back-and-forth. At a stage in the construction we have $\bar{d},[u, v]$ and $\bar{d}^{\prime},\left[u^{\prime}, v^{\prime}\right]$ such that:

1. $\operatorname{tp}(\bar{c}, \bar{d}, u, v)=\operatorname{tp}\left(\bar{c}, \bar{d}^{\prime}, u^{\prime}, v^{\prime}\right)$;

2. $I \in[u, v]$ and $I^{\prime} \in\left[u^{\prime}, v^{\prime}\right]$;

3. $[u, v]$ is $\bar{c}, \bar{d}$-constant and $\bar{c}, \bar{d}$-small and $\left[u^{\prime}, v^{\prime}\right]$ is $\bar{c}, \bar{d}^{\prime}$-constant and $\bar{c}, \bar{d}^{\prime}$ small.

We shall do the 'forth' step extending $\bar{d}$ to $\bar{d}, e$. 'Back' is symmetrical.

Let $h \in \operatorname{Aut}(M, \bar{c})$ with $h(\bar{d})=\bar{d}^{\prime}$ and $h[u, v]=\left[u^{\prime}, v^{\prime}\right]$. Let $e \in M$ be arbitrary and take $I \in\left[u_{0}, v_{0}\right]$ where $\left[u_{0}, v_{0}\right]$ is $\bar{c}, \bar{d}, e, u, v$-constant and $\bar{c}, \bar{d}, e, u, v$ small by genericity of $I$. Then clearly $\left[u_{0}, v_{0}\right] \subseteq[u, v]$ since $\left[u_{0}, v_{0}\right]$ must meet $[u, v]$ as $I$ is contained in both and by $u, v$-constant cannot contain either $u$ or $v$. Consider $h\left[u_{0}, v_{0}\right] \subseteq\left[u^{\prime}, v^{\prime}\right]$. This is $\bar{c}, \bar{d}^{\prime}, h(e)$-constant and $\bar{c}, \bar{d}^{\prime}, h(e)$-small so by the genericity of $I^{\prime}$ there is $k \in \operatorname{Aut}\left(M, \bar{c}, \bar{d}^{\prime}\right)$ and $\left[u_{0}^{\prime}, v_{0}^{\prime}\right] \subseteq\left[u^{\prime}, v^{\prime}\right]$ such that $I^{\prime} \in\left[u_{0}^{\prime}, v_{0}^{\prime}\right]=k h\left[u_{0}, v_{0}\right]$. Let $e^{\prime}=k h(e)$ and continue the construction with $\bar{d}, e,\left[u_{0}, v_{0}\right]$ and $\bar{d}^{\prime}, e^{\prime},\left[u_{0}, v_{0}^{\prime}\right]$ in place of $\bar{d},[u, v]$ and $\bar{d}^{\prime},\left[u^{\prime}, v^{\prime}\right]$.

It is evident that if this construction of an automorphism $g: \bar{d} \mapsto \bar{d}^{\prime}$ is completed in the back-and-forth style we have $g I=I^{\prime}$, as required.

Finally, we prove the existence of generic cuts.

Theorem 5.7. It is enforceable that the cut $I$ is generic.

Proof. We must show how in a Banach-Mazur game we can enforce the resulting cut to be generic.

Given an interval $[u, v]$ and tuple of parameters $\bar{c}$, by Proposition 5.4 followed by Proposition 5.2 we can find $\left[u^{\prime}, v^{\prime}\right] \subseteq[u, v]$ which is $\bar{c}$-small and $\bar{c}$-constant. 
We play such an interval at some stage of the Banach-Mazur game for each tuple $\bar{c}$. This enables us to enforce property (a) in Definition 5.5.

Now suppose we are playing the game, having been given $[u, v]$, and are considering a $\bar{c}$-constant interval $[a, b] \supseteq[u, v]$ and a $\bar{c}$-small interval $[r, s] \subseteq[a, b]$. We want to find $g \in \operatorname{Aut}(M, \bar{c})$ such that $g[r, s] \subseteq[u, v]$ for then we can continue the game playing this interval $g[r, s]$. All such constants $\bar{c}$ and such intervals $[a, b]$ and $[r, s]$ are to be considered in this way at some point in the game.

To achieve our objective, we first choose $t=g(r) \in[u, v]$ such that:

1. for all formulas $\theta(x, \bar{c}), M \vDash \theta(t, \bar{c}) \leftrightarrow \theta(r, \bar{c})$;

2. $Y_{n}(t) \leqslant v$ for all $n \in \mathbb{N}$;

3. $t \geqslant u$.

The choice of this $t$ is straightforward from the recursive saturation of $M$ as it is just realising a type in $M$. In particular, note that the type just written down is finitely satisfied as a consequence of the $\bar{c}$-constant nature of $[a, b]$ and so elements realising the same type as $r$ appear densely in $[a, b]$.

Now we use smallness of $[r, s]$. In particular $[r, s]$ is $\bar{c}$-small, so $s=Y_{n}(r)$ for some nonstandard $n \in M$ with $n<x$ for each $x \in \operatorname{dcl}(r, \bar{c}) \backslash \mathbb{N}$. To define $g(s)$ it suffice to select $m=g(n)$ and put $g(s)=Y_{g(n)}(g(r))=Y_{m}(t)$. First take $n^{\prime}>\mathbb{N}$ such that $Y_{n^{\prime}}(t) \in[u, v]$ and then take $m \leqslant n^{\prime}$ such that $\operatorname{tp}(\bar{c}, t, m)=\operatorname{tp}(\bar{c}, r, n)$. Once again this is by recurive saturation, using the fact that $\operatorname{tp}(\bar{c}, r, n)$ is coded, and that the corresponding type $\operatorname{tp}(\bar{c}, t, m)$ is finitely satisfied by $m<n^{\prime}$ in $M$ : if this last were not the case then for some true $\theta(\bar{c}, r, n)$ we would have the least $m \in M$ such that $\theta(\bar{c}, t, m)$ is greater than $n^{\prime}$, hence the least $m \in M$ such that $\theta(\bar{c}, r, m)$ is a nonstandard element of $\operatorname{dcl}(\bar{c}, r)$ which is greater than $n$, contradicting smallness.

Thus generic cuts enjoy all the properties enforceable in the Banach-Mazur game. For given a generic cut $I$ and any $\bar{c} \in M$ we may take a $\bar{c}$-small $\bar{c}$-constant $[a, b]$ containing $I$, and play the Banach-Mazur game to obtain a generic $I^{\prime} \in$ $[a, b]$ satisfying the property $P$ we are interested in. Then by the above, $I$ and $I^{\prime}$ are conjugate by an automorphism fixing $\bar{c}$ and hence (provided the property $P$ is preserved by all such automorphisms, and all the above properties clearly are) our cut $I$ also has $P$.

A natural question to ask is whether generic cuts exist for $M$ if $M$ is not arithmetically saturated. For example, do generic cuts exist under the assumption that $M$ is recursively saturated? I don't know the answer. There are in fact two versions of this question, one slightly more interesting than the other: firstly, do generic cuts exist, where 'generic' means according to Definition 5.5? and, secondly and slightly more interestingly, is there a notion of generic in the more general setting that includes Definition 5.5 as a special case for which versions of Theorem 5.6 and Theorem 5.7 can be proved?

\section{Symbiosis}

The results in Section 4 in one light are positive, in that they show that a cut with a great number of interesting properties (semiregular, not an $\omega$-limit, all $\Pi_{1}$-types are coded, and so on) can be constructed using an indicator. They 
also, however, show that many properties such as regular or strong, cannot be enforced in such a construction. I cannot see any straightforward topological way out of this dilemma at the level of the generality of this paper.

Paris and Kirby originally constructed strong and regular cuts by means of games [3] and then by combinatorial notions of 'density'. Chapter 4 of Kirby's thesis [2] contains a notion of ' $n$-density' which (for nonstandard $n$ ) suffices to build regular cuts and hence cuts satisfying $\mathrm{B} \Sigma_{2}$, and Paris's original paper on independence results for PA [8] contains a different notion (also called ' $n$-density') that is used to build strong cuts and hence cuts satisfying PA. These notions of density seem to be ad hoc notions specifically designed for the constructions in mind, and are not obviously topological, but are instead combinatorial.

However, using the easier Banach-Mazur constructions above and some further modifications, alternative straightforward constructions of regular and strong cuts can be given. This will be demonstrated below. But first, we turn to a restricted version of Question 2.1. This restriction to local properties is, we think, natural and very mild.

Definition 6.1. A property $P^{M}(I)$ of a proper cut $I \subseteq$ e $M$ is local if whenever $I \subseteq \mathrm{e} J \subseteq \mathrm{e} M$ and $I \neq J$ then

$$
P^{M}(I) \Leftrightarrow P^{J}(I)
$$

More generally, $P^{M}(I)$ is $Y$-local if $P^{M}(I) \Leftrightarrow P^{J}(I)$ whenever $I \subseteq$ e $J, I \neq J$ and $P_{Y}^{M}(I), P_{Y}^{M}(J)$ both hold.

For example, denoting by $\mathrm{SSy}_{I}(M)$ the set of subsets of $I$ coded in $M$,

$$
\operatorname{SSy}_{I}(M)=\left\{A \subseteq I \mid \exists a \in M A=\left\{i \in I \mid M \vDash(a)_{i} \neq 0\right\}\right\},
$$

then any property $P^{M}(I)$ depending on $I$ and $\operatorname{SSy}_{I}(M)$ only is local as $\operatorname{SSy}_{I}(J)=$ $\operatorname{SSy}_{I}(M)$. All the usual combinatorial properties, such as 'strong', 'regular', etc., are local for this reason.

The next result gives model-theoretic description of the local properties of indicated cuts. The saturation assumptions on $I$ here (that $\Pi_{1}-\operatorname{Th}(M, a)$ is coded, $I$ is not an $\omega$-limit, and $I$ is short $\Pi_{1}$-recursively saturated) are convenient, being the ones most useful in practice, and are made available by previous results. The exact details of these conditions are not particularly essential: they could be replaced by ' $I$ is generic' for example. We emphasise that in this and all other results in this section there is a global assumption that $M$ is countable.

Theorem 6.2. An indicator $Y$ indicates cuts satisfying a local property $P^{M}(I)$ if and only if for all $a \in I \in Z_{Y}$ with $\Pi_{1}-\operatorname{Th}(M, a)$ coded, $I$ not an $\omega$-limit, and $I$ short $\Pi_{1}$-recursively saturated there is $I \subseteq_{\mathrm{e}} J \subseteq_{\mathrm{e}} K \vDash \Pi_{1}-\mathrm{Th}(M, a)+\mathrm{B} \Sigma_{1}$ such that $J \neq K, K$ is short $\Pi_{1}$-recursively saturated and $P^{K}(J)$.

Proof. For one direction, suppose $Y$ indicates cuts satisfying $P^{M}(I)$, and suppose $a \in I \in Z_{Y}$ with $\Pi_{1}-\operatorname{Th}(M, a)$ coded, $I$ not an $\omega$-limit, and $I$ short $\Pi_{1}$-recursively saturated. Then let $c>I$ and $b_{n}$ be the greatest $b<c$ such that $M \vDash \forall x<b \theta(x, a)$ for all $\theta(x, a)$ with Gödel-number at most $n$ with $I \vDash \forall x \theta(x, a)$. By $\Sigma_{2}$-recursive saturation of $M$ the sequence $b_{n}$ is coded, and as $I$ is not an $\omega$-limit there is $b>I$ in $M$ such that $b<b_{n}$ for each $n$. 
So $Y(a, b)>\mathbb{N}$. Again using our assumption that $I$ is not an $\omega$-limit, there is $I<c<b$ with $Y(c, b)>\mathbb{N}$ since the maximum $c_{n}$ such that $Y\left(c_{n}, b\right)>n$ is above $I$ and the sequence of such $c_{n}$ is coded and does not tend to $I$. Hence there is $d \in M$ with $Y(c, d)>\mathbb{N}$ and $Y(d, b)>\mathbb{N}$ and there is some $K \in Z_{Y}$ with $d \in K<b$ and by a Banach-Mazur game and the results of Section 4 we may take $K$ to be short $\Pi_{1}$-recursively saturated. Also there is some $J \subseteq_{\mathrm{e}} K$ with $c \in J<d$ and $P^{M}(J)$. Thus $P^{K}(J)$ as $P$ is local, and as $K<b$ we have $K \vDash \Pi_{1}-\operatorname{Th}(M, a)+\mathrm{B} \Sigma_{1}$, so we have $I \subseteq J \subseteq K \vDash \Pi_{1}-\operatorname{Th}(M, a)+\mathrm{B} \Sigma_{1}$ such that $P^{K}(J)$ as required.

Conversely, suppose there are end-extensions $J, K$ as described for each suitable $a \in I \in Z_{Y}$. Then suppose $Y(a, b)>\mathbb{N}$. We must show there is $a \in I<b$ with $P^{M}(I)$. Take instead some $I \in Z_{Y}$ with $a \in I<b$ and $\Pi_{1}-\operatorname{Th}(M, a)$ coded, $I$ not an $\omega$-limit and $I$ short $\Pi_{1}$-recursively saturated; such $I$ exists by a Banach-Mazur game and the results of Section 4 . Then by hypothesis there are $I \subseteq$ e $J \subseteq$ e $K \vDash \Pi_{1}-\operatorname{Th}(M, a)+\mathrm{B} \Sigma_{1}$ such that $P^{K}(J)$ and $K$ is short $\Pi_{1}$-recursively saturated. By Friedman's embedding theorem $[1$, Chapter 12] and $K \vDash \Pi_{1}-\operatorname{Th}(M, a)$ there is an embedding $h$ of $K$ onto an initial segment $h(K)<b$ of $M$, where $h(a)=a$. Then $a \in J$ and so $a=h(a) \in h(J) \subseteq \mathrm{e} h(K)<b$ hence $h(J)$ has $P^{h(K)}(h(J))$, so $h(J)$ has the property $P^{M}(h(J))$ as $P$ is local. Thus $Y$ indicates the property $P$.

Definition 6.3 (Kirby-Paris). Two properties $P(I)$ and $Q(I)$ of initial segments $I$ are symbiotic if whenever $a<b \in M$ then: there is $I \subseteq$ e $M$ with $a \in I<b$ and $P(I)$ if and only if there is $J \subseteq_{\mathrm{e}} M$ with $a \in J<b$ and $Q(J)$.

As an application of the ideas already presented, let us sketch new proofs of two old results of Kirby and Paris.

Corollary 6.4. The properties ' $I$ is strong' and ' $I \vDash \Pi_{2}-\mathrm{Th}(\mathrm{PA})$ ' are symbiotic.

Proof. We take any indicator $Y$ for the $\Pi_{2}$-consequences of $\mathrm{PA}$; such indicators can be constructed directly [1, Chapter 14] or we may base $Y$ on the ParisHarrington statement. Thus, for all initial segments $I$ of $M$, we have $I \in Z_{Y}$ iff $I \vDash \Pi_{2}-\mathrm{Th}(\mathrm{PA})$. Since ' $I$ is strong' implies ' $I \vDash \mathrm{PA}$ ' [3] we simply have to show that there is a strong $I \in(a, b)_{Y}$ whenever $(a, b)_{Y} \neq \emptyset$.

Let $a \in I<b$ where $I \in Z_{Y}$; by Section 4 we may assume that $I$ satisfies the conditions in Theorem 6.2. Since $I$ satisfies $\Pi_{2}-\operatorname{Th}(\mathrm{PA})$ and $\Pi_{1}-\operatorname{Th}(I, a)$ we have $I \vDash \Pi_{1}-\mathrm{Th}\left(\mathrm{PA}+\Pi_{1}-\mathrm{Th}(I, a)\right)$ for if $\mathrm{PA}+\sigma(a) \vdash \tau(a)$ with $I \vDash \sigma(a)$ and $\tau(a), \sigma(a)$ both $\Pi_{1}$ then $\mathrm{PA} \vdash \forall x(\sigma(x) \rightarrow \tau(x))$ which is $\Pi_{2}$, hence $I \vDash \tau(a)$. Since $I \vDash B \Sigma_{1}$, is short $\Pi_{1}$-recursively saturated and the required theory is coded, there is $J \supseteq_{\mathrm{e}} I$ with $J \models \mathrm{PA}+\Pi_{1}-\mathrm{Th}(I, a)$.

Now let $K \supseteq \mathrm{e} J$ with $K \vDash \mathrm{B} \Sigma_{1}+\Pi_{1}-\mathrm{Th}(I, a)$ be constructed by means of the arithmetised completeness theorem. Such a $K$ exists as $\Pi_{1}-\operatorname{Th}(I, a)$ is coded and consistent, so by the satisfaction relation for $\Pi_{1}$ formulas in $J$ and an induction, $J \vDash \operatorname{Con}\left(\mathrm{I} \Delta_{0}+T(a)\right)$ for some $J$-finite $\Pi_{1}$ set of formulas $T(a) \supseteq \Pi_{1}-\operatorname{Th}(I, a)$. But PA proves that $\mathrm{B} \Sigma_{1}$ is $\Pi_{2}$-conservative over $\mathrm{I} \Delta_{0}$ hence $J \vDash \operatorname{Con}\left(\mathrm{B} \Sigma_{1}+T(a)\right)$ and so $K$ exists by the arithmetised completeness theorem. As $K$ is an arithmetised completeness theorem model constructed from the nonstandard model $J$ it is recursively saturated and hence short $\Pi_{1}$ recursively saturated. 
Now it is straightfoward to use $J \vDash \mathrm{PA}$ to see that $\left(J, \operatorname{SSy}_{J}(K)\right) \vDash \mathrm{PA}$ in this expanded language, or - in the notation of Kirby and Paris [3]- $J \vDash \mathrm{PA}^{*}$. This is because any subset of $J$ coded in $K$ is actually represented by a $J$-finite formula of the language of arithmetic and this in turn can be used to give a direct $\Delta_{1}$ definition of the subset in $J$. The property $J \vDash \mathrm{PA}^{*}$ is, by an alternative characterisation of 'strong' due to Kirby and Paris, equivalent to saying that $J$ is strong in $K$, and we have verified the conditions of Theorem 6.2.

Corollary 6.5. The properties ' $I$ is regular' and ' $I \vDash \Pi_{2}-\operatorname{Th}\left(\mathrm{I} \Sigma_{1}\right)$ ' are symbiotic.

Proof. Our indicator now is $Y(x, y)=\max \left\{n \mid F_{n}(x) \leqslant y\right\}$ where $F_{n}$ is the Grzegorczyk hierarchy of functions; this indicator is absolute in the sense of Definition 2.8 and $\mathrm{I} \Delta_{0}$ together with the axiom scheme $\forall x \exists y F_{n}(x)=y$ for $n \in \mathbb{N}$ axiomatizes $\Pi_{2}-\operatorname{Th}\left(\mathrm{I} \Sigma_{1}\right)$. Any cut $I \in(a, b)_{Y}$ satisfies $\Pi_{2}-\operatorname{Th}\left(\mathrm{I} \Sigma_{1}\right)$. In other respects the argument is very similar to the preceding one.

Let $a \in I \vDash \mathrm{B} \Sigma_{1}+\Pi_{2}-\mathrm{Th}\left(\mathrm{I} \Sigma_{1}\right)$ satisfy the conditions in Theorem 6.2. By saturation, the fact that $\mathrm{B} \Sigma_{2}$ is $\Pi_{2}$-conservative over $\mathrm{I} \Sigma_{1}[1$, Chapter 10] and the fact that $\Pi_{1}-\operatorname{Th}(I, a)$ is coded in $I$, there is an end-extension $J \supseteq_{\mathrm{e}} I$ of $I$ with $J \models \mathrm{B} \Sigma_{2}+\Pi_{1}-\mathrm{Th}(I, a)$. By the arithmetized completeness theorem there is an end-extension $K \supseteq_{\mathrm{e}} J$ of $J$ with $K \vDash \mathrm{B} \Sigma_{1}+\Pi_{1}-\operatorname{Th}(I, a)$. Now using $J \vDash \mathrm{B} \Sigma_{2}$ we can see that $\left(J, \operatorname{SSy}_{J}(K)\right) \vDash \mathrm{B} \Sigma_{2}$ in the expanded language, i.e., $J \models \mathrm{B} \Sigma_{2}^{*}$. This is again because each $K$-coded subset of $J$ is definable in $J$ by a $\Delta_{1}$ formula. This $\mathrm{B} \Sigma_{2}^{*}$ is an alternative characterisation of 'regular'. Thus, by Kirby and Paris [3], $J$ is regular in $K$ and Theorem 6.2 is satisfied.

We finish this paper by stating a conjecture for the preservation theorem question. The most natural class of properties $P^{M}(I)$ to understand are firstorder properties of the expanded structure $\left(I, \operatorname{SSy}_{I}(M)\right)$.

Conjecture 6.6. The first-order properties of the form $\left(I, \operatorname{SSy}_{I}(M)\right) \vDash \bigwedge_{i \in \mathbb{N}} \theta_{i}$ that have an indicator $Y$ uniformly for all countable models $M \vDash$ PA are precisely those properties that can be re-written as $\left(I, \operatorname{SSy}_{I}(M)\right) \vDash \bigwedge_{i \in \mathbb{N}} \psi_{i}$ with $\left\{\psi_{i} \mid i \in \mathbb{N}\right\}$ recursive.

The other important class of properties $P^{M}(I)$ of a cut are $\Sigma_{1}^{1}$ properties, so that the property looks like $\left(I, \operatorname{SSy}_{I}(M)\right) \vDash \exists R, f, \ldots \bigwedge_{i \in \mathbb{N}} \theta_{i}(R, f, \ldots)$. Forexample, it is possible to say that $I$ is recursively saturated in such a way. For this we would make a similar conjecture, that the class of such properties with an indicator (uniformly in all countable models of PA) are those for which the $\Sigma_{1}^{1}$ formula $\exists R, f, \ldots \bigwedge_{i \in \mathbb{N}} \theta_{i}(R, f, \ldots)$ is equivalent to a recursive one.

\section{Acknowledgements}

The author would like to thank Costas Dimitracopoulos, Roman Kossak, Henryk Kotlarski, Alexandra Sirokofski and Tin Lok Wong for many stimulating conversations while this paper was being written, and the anonymous referees for very helpful comments regarding the finished version. 


\section{References}

[1] Richard Kaye. Models of Peano arithmetic. The Clarendon Press Oxford University Press, New York, 1991. Oxford Science Publications.

[2] L. A. S. Kirby. Initial segments of models of arithetic. Ph.D., Manchester University, July 1977.

[3] L. A. S. Kirby and J. B. Paris. Initial segments of models of Peano's axioms. In Set theory and hierarchy theory, V (Proc. Third Conf., Bierutowice, 1976), pages 211-226. Lecture Notes in Math., Vol. 619. Springer, Berlin, 1977.

[4] Roman Kossak and James H. Schmerl. The structure of models of Peano arithmetic, volume 50 of Oxford Logic Guides. The Clarendon Press Oxford University Press, Oxford, 2006. Oxford Science Publications.

[5] Henryk Kotlarski. Some remarks on initial segments in models of Peano arithmetic. J. Symbolic Logic, 49(3):955-960, 1984.

[6] Daniel Lascar. The small index property and recursively saturated models of Peano arithmetic. In Automorphisms of first-order structures, Oxford Sci. Publ., pages 281-292. Oxford Univ. Press, New York, 1994.

[7] John C. Oxtoby. Measure and category, volume 2 of Graduate Texts in Mathematics. Springer-Verlag, New York, 1971.

[8] J. B. Paris. Some independence results for Peano arithmetic. J. Symbolic Logic, 43(4):725-731, 1978.

[9] J. B. Paris and L. A. S. Kirby. $\Sigma_{n}$-collection schemas in arithmetic. In Logic Colloquium 'ry (Proc. Conf., Wroctaw, 1977), pages 199-209. NorthHolland, Amsterdam, 1978.

[10] Jeff Paris and Leo Harrington. A mathematical incompleteness in Peano arithmetic. In A Handbook of Mathematical Logic, pages 1133-1142. North Holland, 1977.

[11] A. Wilkie and J. Paris. On the existence of end extensions of models of bounded induction. In Logic, methodology and philosophy of science, VIII (Moscow, 1987), volume 126 of Stud. Logic Found. Math., pages 143-161. North-Holland, Amsterdam, 1989. 